\title{
ON COMPARABILITY RELATIONS IN THE CLASS OF INTERVAL-VALUED FUZZY RELATIONS
}

\author{
Barbara Peskala - Urgzula Bentkowska - Bernard De Baets
}

\begin{abstract}
In this paper, a new relation for the set of interval-valued fuzzy relations is introduced. This relation is an interval order for the family of intervals and for the family of interval-valued fuzzy relations in a given set, it has the reflexivity property. Consequences of considering such a relation are studied in the context of operations on interval-valued fuzzy relations. A new transitivity property, namely possible $T$-transitivity is studied (pos- $T$-transitivity for short). This transitivity property is connected with the new relation proposed in this paper. Preservation of this type of transitivity by some operations is also discussed.
\end{abstract}

\section{Introduction}

L. A. Z a d e h [17] introduced interval-valued fuzzy relations as a generalization of the concept of fuzzy relations [16]. Interval valued fuzzy sets and relations have applications in diverse types of areas, for example in classification, image processing and multicriteria decision making.

A comparative study of the existing definitions of order relations between intervals, analyzing the level of acceptability and shortcomings from different points of view was presented in [13. Orders used for interval-valued fuzzy relations may be connected with ontic and epistemic setting [5], 6]. Epistemic uncertainty represents the idea of partial or incomplete information. It may be described by means of a set of possible values of some quantity of interest, one of which is the right one. A fuzzy set represents in such an approach incomplete information, so it may be called disjunctive [5]. On the other hand, fuzzy

(C) 2016 Mathematical Institute, Slovak Academy of Sciences.

2010 Mathematics Subject Classification: 03E72, 68T37.

Keywords: partial order, interval order, $T$-transitivity, pos- $T$-transitivity, interval-valued fuzzy relation.

Partially supported by the Centre for Innovation and Transfer of Natural Sciences and Engineering Knowledge in Rzeszów, through the Project Number RPPK.01.03.00-18-001/10. 
sets may be conjunctive and can be called ontic fuzzy sets [5]. In this situation, the fuzzy set is used for representing some precise gradual entity consisting of a collection of items.

The aim of this work is to examine dependencies between the natural order (which is a partial order) and here introduced relation in the set of interval-valued fuzzy relations. Moreover, consequences of considering such a relation are studied in the context of operations on interval-valued fuzzy relations, among others the new transitivity property called possible $T$-transitivity (pos- $T$-transitivity) is discussed.

The paper is structured as follows. Firstly, some concepts and results useful in further considerations are recalled (Section 2). Next, results connected with the interval order are presented (Section 3). Moreover, some properties of operations on interval-valued fuzzy relations are studied (Section 4). In Section 5 , the pos- $T$-transitivity, based on the definition of the given new relation, is presented and its preservation by basic operations is considered.

\section{Properties of interval-valued fuzzy relations}

We recall definition of the lattice operations and the order for interval-valued fuzzy relations.

\subsection{Structure of interval-valued fuzzy relations}

Definition 2.1 (cf. [15], 17]). Let $X, Y$ be non-empty sets. An interval-valued fuzzy relation $R$ between universes $X, Y$ is a mapping $R: X \times Y \rightarrow L^{I}$ such that

$$
R(x, y)=[\underline{R}(x, y), \bar{R}(x, y)] \in L^{I},
$$

for all couples $(x, y) \in(X \times Y)$, where $L^{I}=\left\{\left[x_{1}, x_{2}\right]: x_{1}, x_{2} \in[0,1], x_{1} \leq x_{2}\right\}$.

The class of all interval-valued fuzzy relations between universes $X, Y$ will be denoted by $\mathcal{I} \mathcal{V} \mathcal{F} \mathcal{R}(X \times Y)$ or $\mathcal{I} \mathcal{V} \mathcal{F} \mathcal{R}(X)$ for $X=Y$.

We use the following partial order for intervals:

$$
\left[x_{1}, y_{1}\right] \leq\left[x_{2}, y_{2}\right] \Leftrightarrow x_{1} \leq x_{2}, y_{1} \leq y_{2} .
$$

For every $(x, y) \in(X \times Y), P=[\underline{P}, \bar{P}], R=[\underline{R}, \bar{R}] \in I V F R(X)$ we have

$$
P(x, y) \leq R(x, y) \Leftrightarrow \underline{P}(x, y) \leq \underline{R}(x, y), \bar{P}(x, y) \leq \bar{R}(x, y) .
$$

The boundary elements in $\mathcal{I} \mathcal{V} \mathcal{F} \mathcal{R}(X \times Y)$ are $\mathbf{1}=[1,1]$ and $\mathbf{0}=[0,0]$. Let $P, R \in \mathcal{I} \mathcal{V} \mathcal{F} \mathcal{R}(X \times Y)$, then

$$
\begin{aligned}
& (P \vee R)(x, y)=[\max (\underline{P}(x, y), \underline{R}(x, y)), \max (\bar{P}(x, y), \bar{R}(x, y))], \\
& (P \wedge R)(x, y)=[\min (\underline{P}(x, y), \underline{R}(x, y)), \min (\bar{P}(x, y), \bar{R}(x, y))] .
\end{aligned}
$$




\section{COMPARABILITY RELATIONS IN CLASS OF INTERVAL-VALUED FUZZY RELATIONS}

The structure $(\mathcal{I} \mathcal{V} \mathcal{F} \mathcal{R}(X \times Y), \leq)$ is a partially ordered set, i.e., the relation $\leq$ is:

- reflexive, $R(x, y) \leq R(x, y)$,

- antisymmetric, $R(x, y) \leq P(x, y)$ and $P(x, y) \leq R(x, y) \Rightarrow R(x, y)=P(x, y)$,

- transitive, $R(x, y) \leq P(x, y)$ and $P(x, y) \leq Q(x, y) \Rightarrow R(x, y) \leq Q(x, y)$ for every $(x, y) \in(X \times Y)$ and $P, Q, R \in \mathcal{I} \mathcal{V} \mathcal{F} \mathcal{R}(X \times Y)$.

For an arbitrary index set $D \neq \emptyset$ it holds that

$$
\begin{aligned}
& \left(\bigvee_{d \in D} R_{d}\right)(x, y)=\left[\sup _{d \in D} \frac{R_{d}}{(x, y)}, \sup _{d \in D} \overline{R_{d}}(x, y)\right]
\end{aligned}
$$

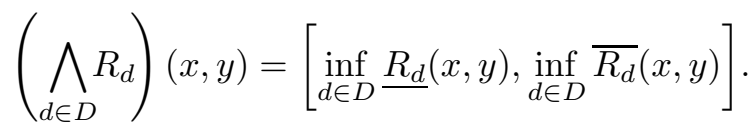

More general classes of operations are triangular norms.

Definition $2.2([2])$. A triangular norm $\mathbf{T}$ on a bounded poset $\mathbf{P}=(\mathbf{P}, \leq, 0,1)$ is an increasing commutative associative operation $\mathbf{T}: \mathbf{P}^{\mathbf{2}} \rightarrow \mathbf{P}$ with a neutral element 1.

One of the possible construction methods for triangular norms is representability. An operation $\mathcal{T}:\left(L^{I}\right)^{2} \rightarrow L^{I}$ is called a representable triangular norm if there exist triangular norms $T_{1}, T_{2}:[0,1]^{2} \rightarrow[0,1]$ such that for all $x=\left[x_{1}, x_{2}\right], y=$ $\left[y_{1}, y_{2}\right] \in L^{I}$ and $T_{1} \leq T_{2}$ :

$$
\mathcal{T}(x, y)=\left[T_{1}\left(x_{1}, y_{1}\right), T_{2}\left(x_{2}, y_{2}\right)\right] .
$$

\subsection{Interval order in the class $L^{I}$}

Now, we recall definition of an interval order for crisp relations. The name "interval order" first appeared in F i s h b u r n [7]-[9].

Definition 2.3 ([10, p. 42]). A relation $R \subset X \times X$ is an interval order if it is complete and has the Ferrers property, i.e.:

- $R(x, y)$ or $R(y, x)$, for $x, y \in X$,

- $R(x, y)$ and $R(z, w) \Rightarrow R(x, w)$ or $R(z, y)$, for $x, y, z, w \in X$, respectively.

We will consider the following relation between intervals in $L^{I}$ :

$$
\left[x_{1}, y_{1}\right] \preceq\left[x_{2}, y_{2}\right] \Leftrightarrow x_{1} \leq y_{2} .
$$

Proposition 2.4. In the structure $\left(L^{I}, \preceq\right)$, the relation $\preceq$ is an interval order.

P r o o f. Let $\left[a_{1}, b_{1}\right] \preceq\left[a_{2}, b_{2}\right]$ and $\left[a_{3}, b_{3}\right] \preceq\left[a_{4}, b_{4}\right]$ for $\left[a_{i}, b_{i}\right] \in L^{I}, i \in\{1,2,3,4\}$, so $a_{1} \leq b_{2}, a_{3} \leq b_{4}$.

- If $a_{1}>b_{4}$, then $a_{3} \leq a_{1}$, i.e., $a_{3} \leq b_{2}$.

- If $a_{3}>b_{2}$, then $a_{1} \leq a_{3}$, i.e., $a_{1} \leq b_{4}$. 
As a result, relation $\preceq$ has the Ferrers property, i.e.,

$$
\begin{aligned}
& {\left[a_{1}, b_{1}\right] \preceq\left[a_{2}, b_{2}\right] \quad \text { and } \quad } {\left[a_{3}, b_{3}\right] \preceq\left[a_{4}, b_{4}\right] \Rightarrow\left[a_{1}, b_{1}\right] \preceq\left[a_{4}, b_{4}\right] } \\
& \text { or } \quad\left[a_{3}, b_{3}\right] \preceq\left[a_{2}, b_{2}\right] .
\end{aligned}
$$

- If $a_{1} \leq b_{2}$, then $\left[a_{1}, b_{1}\right] \preceq\left[a_{2}, b_{2}\right]$.

- If $a_{1} \geq b_{2}$, then $\left[a_{2}, b_{2}\right] \preceq\left[a_{1}, b_{1}\right]$.

As a result, relation $\preceq$ is complete, i.e.,

$$
\left[a_{1}, b_{1}\right] \preceq\left[a_{2}, b_{2}\right] \quad \text { or } \quad\left[a_{2}, b_{2}\right] \preceq\left[a_{1}, b_{1}\right] .
$$

The following connection between the natural (partial) order $\leq$ and the interval order $\preceq$ follows from (11) and (2).

COROLLARY 2.5. If the natural order (11) holds, then also the interval order (2) holds.

The converse implication does not hold, as can be seen from the following example.

ExAmple 2.6. For intervals $A=[0.2,0.8]$ and $B=[0.1,1]$ we observe that $A \preceq B$ but it is not true that $A \leq B$.

We would like to use this new comparability relation in the class $\mathcal{I} \mathcal{V} \mathcal{F} \mathcal{R}(X \times Y)$ and examine the consequences of this choice. Thus, for every $(x, y) \in(X \times Y)$, $P=[\underline{P}, \bar{P}], R=[\underline{R}, \bar{R}] \in \mathcal{I} \mathcal{V} \mathcal{F} \mathcal{R}(X \times Y)$ we have

$$
P(x, y) \preceq R(x, y) \Leftrightarrow \underline{P}(x, y) \leq \bar{R}(x, y) .
$$

Relation $\preceq$ is more suitable for the epistemic setting of the interval-valued fuzzy relations. Namely, if $\left[x_{1}, y_{1}\right]$ is an unprecise description of a variable $x$ and $\left[x_{2}, y_{2}\right]$ is an unprecise description of a variable $y$, then $\left[x_{1}, y_{1}\right] \preceq\left[x_{2}, y_{2}\right]$ denotes that it is possible that the true value of $x$ is smaller than or equal to the true value of $y$. The relation $\preceq$ thus has a possibilistic interpretation [4]. We can write

$$
\exists_{P^{*}} \exists_{R^{*}} P \preceq R \Leftrightarrow \underline{P} \leq \bar{R}
$$

and we may call this relation possible comparability relation of $R$ and $P$.

Let us notice that the relation $\preceq$ in the family $\mathcal{I} \mathcal{V} \mathcal{F} \mathcal{R}(X \times Y)$ has the reflexivity property, i.e., $R \preceq R$. However, it is not complete and does not have the Ferrers property. As a result, it is not an interval order relation in $\mathcal{I} \mathcal{V} \mathcal{F} \mathcal{R}(X \times Y)$.

ExAmple 2.7. For $R=[[0.1,0.2][0.5,0.6]]$ and $S=[[0.3,0.4][0.1,0.4]]$ we see that $\sim(R \preceq S)$ and $\sim(S \preceq R)$. Similarly, we can show the lack of the Ferrers property. 


\section{Properties of operations in $(\mathcal{I V \mathcal { F } R}(X \times Y), \preceq)$}

Firstly, we consider connections between basic operations on $\mathcal{I} \mathcal{V F R}(X \times Y)$ and the considered relation $\preceq$. For $(x, y) \in(X \times Y), P=[\underline{P}, \bar{P}], R=[\underline{R}, \bar{R}] \in$ $\mathcal{I V} \mathcal{F} \mathcal{R}(X \times Y)$ we have

$$
\begin{aligned}
& P \wedge R \preceq P \preceq P \vee R, \\
& P \wedge R \preceq R \preceq P \vee R .
\end{aligned}
$$

Moreover, if $\bar{P} \geq \bar{R}$, then $R \preceq P \wedge R$ and if $\bar{P} \leq \bar{R}$, then $P \preceq P \wedge R$. Interesting differences between the considered relation $\preceq$ and the natural (partial) order present the following conditions

and

$$
P \preceq R \Leftarrow(P \wedge R=P, P \vee R=R)
$$

$$
\text { if } \bar{P} \leq \underline{R} \text {, then } P \preceq R \Rightarrow(P \wedge R=P, P \vee R=R) .
$$

Moreover, we have the implication

$$
(R \preceq P, P \preceq R) \Leftarrow(\underline{R}=\underline{P}, \bar{R}=\bar{P}),
$$

but the converse implication we obtain if the relation $\preceq$ is replaced with the natural order $\leq$. If we consider the converse operation $R^{t}(x, y)=R(y, x)$, then it holds

$$
P \preceq R \Leftrightarrow P^{t} \preceq R^{t} .
$$

Other interesting properties for considered here relation $\preceq$, we present in the following result.

Proposition 3.1. Let $(x, y) \in(X \times Y), P=[\underline{P}, \bar{P}], Q=[\underline{Q}, \bar{Q}], R=[\underline{R}, \bar{R}] \in$ $\operatorname{IVF\mathcal {R}}(X \times Y)$. Then we have

- $P(x, y) \preceq R(x, y), P(x, y) \preceq Q(x, y) \Leftrightarrow P(x, y) \preceq R(x, y) \wedge Q(x, y)$,

- $R(x, y) \preceq P(x, y), Q(x, y) \preceq P(x, y) \Leftrightarrow R(x, y) \vee Q(x, y) \preceq P(x, y)$,

- $P(x, y) \preceq R(x, y)$ and $W(x, y) \preceq Q(x, y) \Rightarrow P(x, y) \vee W(x, y) \preceq$ $R(x, y) \vee Q(x, y)$ and $P(x, y) \wedge W(x, y) \preceq R(x, y) \wedge Q(x, y)$.

P ro of. Let $P(x, y) \preceq R(x, y)$ and $P(x, y) \preceq Q(x, y)$, so $\underline{P} \leq \bar{R}$ and $\underline{P} \leq \bar{Q}$, then $\underline{P} \leq \bar{R} \wedge \bar{Q}$ because $\wedge$ is the infimum in the lattice $([0,1], \wedge, \vee)$. Similarly, we obtain the second condition by the property of supremum $\vee$. Moreover, by isotonicity of these operations we obtain the third condition.

Let us now recall the notion of the composition for interval-valued fuzzy relations. 
Definition 3.2 (cf. [1, [11). Let $P \in \operatorname{IVF\mathcal {R}}(X \times Y), R \in \mathcal{I V F \mathcal { R }}(Y \times Z)$, $\mathbf{T}: L^{I} \times L^{I} \rightarrow L^{I}$ be a triangular norm. The sup $-\mathbf{T}$ composition of the relations $P$ and $R$ is called the relation $P \circ R \in \mathcal{I V F \mathcal { R }}(X \times Z)$,

$$
(P \circ R)(x, z)=\bigvee_{y \in Y} \mathbf{T}(P(x, y), R(y, z)) \text {. }
$$

Especially, if $\mathbf{T}$ is a representable triangular norm $\mathcal{T}$ we have sup $-T_{1} T_{2}$ composition,

where $T_{1} \leq T_{2}$ and

$$
(P \circ R)(x, z)=\left[\left(\underline{P}_{{ }}{ }_{T_{1}} \underline{R}\right)(x, z),\left(\bar{P} \circ_{T_{2}} \bar{R}\right)(x, z)\right],
$$

$$
\begin{aligned}
& \left(\underline{P} \circ_{T_{1}} \underline{R}\right)(x, z)=\sup _{y \in Y} T_{1}(\underline{P}(x, y), \underline{R}(y, z)), \\
& \left(\bar{P} \circ_{T_{2}} \bar{R}\right)(x, z)=\sup _{y \in Y} T_{2}(\bar{P}(x, y), \bar{R}(y, z)) .
\end{aligned}
$$

In our further considerations in the whole paper, we will use the composition with a representable triangular norm and the symbol $\circ$ will mean sup $-T_{1} T_{2}$ composition. For simplicity of notations, we present the results for composition in the class $\mathcal{I} \mathcal{V} \mathcal{F} \mathcal{R}(X)$.

TheOREM 3.3. If $T_{1}, T_{2}, T_{1} \leq T_{2}$ are triangular norms, then

$$
\begin{gathered}
P \preceq R \Rightarrow P \circ Q \preceq R \circ Q, \quad Q \circ P \preceq Q \circ R, \\
P \circ(Q \vee R)=P \circ Q \vee P \circ R .
\end{gathered}
$$

Moreover, if $T_{1}, T_{2}, T_{1} \leq T_{2}$ are supremum preserving then

$$
P \circ(Q \circ R)=(P \circ Q) \circ R \text {. }
$$

P r o o f. Let $P \preceq R$, i.e., $\underline{P} \leq \bar{R}$ and $T_{1} \leq T_{2}$, then by Proposition 3.1 we have for $x, y \in X$

$$
\begin{aligned}
\bigvee_{z \in X} T_{1}(\underline{P}(x, z), \underline{Q}(z, y)) & \leq \bigvee_{z \in X} T_{2}(\bar{R}(x, z), \underline{Q}(z, y)) \\
& \leq \bigvee_{z \in X} T_{2}(\bar{R}(x, z), \bar{Q}(z, y)),
\end{aligned}
$$

so $P \circ Q \preceq R \circ Q$. The second inequality in the first condition can be proven similarly. By distributivity of a triangular norm with respect to maximum we obtain the second condition. Moreover, since triangular norms $T_{1}, T_{2}$ are supremum preserving, we have associativity.

In a semigroup $(\mathcal{I} \mathcal{V} \mathcal{F} \mathcal{R}(X), \circ)$ we can consider the powers of its elements, i.e., relations $R^{n}$ for $R \in \mathcal{I} \mathcal{V} \mathcal{F} \mathcal{R}(X), n \in \mathbb{N}$.

Definition 3.4. Let $R \in \mathcal{I V \mathcal { F } R}(X)$. The powers of $R$ are defined in the following way

$$
R^{1}=R, \quad R^{n+1}=R^{n} \circ R, \quad n \in \mathbb{N} .
$$


The upper operation $R^{\vee}$ and the lower operation $R^{\wedge}$ of $R$ are defined in the following way

$$
R^{\vee}=\bigvee_{k=1}^{\infty} R^{k}, \quad R^{\wedge}=\bigwedge_{k=1}^{\infty} R^{k},
$$

where $R^{k}=\left[\underline{R}^{k}, \bar{R}^{k}\right]$. Now we will examine connections between powers and upper (lower) operations and operations $\vee$ and $\wedge$.

TheOREM 3.5. Let $T_{1}, T_{2}, T_{1} \leq T_{2}$ be supremum preserving triangular norms and $P, R \in \mathcal{I} \mathcal{V} \mathcal{F} \mathcal{R}(X)$.

$$
\text { If } \quad R \preceq P, \quad \text { then } \quad R^{n} \preceq P^{n}, \quad R^{\vee} \preceq P^{\vee}, \quad R^{\wedge} \preceq P^{\wedge}, \quad n \in \mathbb{N} .
$$

Moreover,

$$
\begin{aligned}
& (P \vee R)^{\vee} \succeq P^{\vee} \vee R^{\vee}, \\
& (P \wedge R)^{\vee} \preceq P^{\vee} \wedge R^{\vee}, \\
& (P \vee R)^{\wedge} \succeq P^{\wedge} \vee R^{\wedge}, \\
& (P \wedge R)^{\wedge} \preceq P^{\wedge} \wedge R^{\wedge} .
\end{aligned}
$$

P r o of. By isotonicity of composition we obtain isotonicity of powers, moreover by isotonicity of supremum and infimum we have dependencies for lower and upper operations. By Proposition 3.1 and the above conditions we obtain the rest of results.

\section{Possible T-transitivity}

Many authors, for example in [12, [14, used the following definition of transitivity.

DeFinition 4.1. Let $\mathcal{T}=\left[T_{1}, T_{2}\right]$ be a representable triangular norm and $T_{1} \leq T_{2}$, $R \in \mathcal{I} \mathcal{V} \mathcal{F} \mathcal{R}(X)$. Relation $R$ is called $\mathcal{T}$-transitive if

and

$$
T_{1}(\underline{R}(x, y), \underline{R}(y, z)) \leq \underline{R}(x, z)
$$

$$
T_{2}(\bar{R}(x, y), \bar{R}(y, z)) \leq \bar{R}(x, z) .
$$

Now we will consider the transitivity property connected with the introduced relation $\preceq$ in the epistemic setting. This definition of transitivity naturally follows from the introduced relation $\preceq$, namely replacing the natural order $\leq$ with the relation $\preceq$ we get by Definition 4.1 for a representable triangular norm $\mathcal{T}$ the formula $\mathcal{T}(R(x, y), R(y, z)) \preceq R(x, z)$. As a result, applying definition of the relation $\preceq$ we get the following notion. 


\section{BARBARA PẸKALA — URSZULA BENTKOWSKA - BERNARD DE BAETS}

Definition $4.2([3])$. Let $T:[0,1]^{2} \rightarrow[0,1]$ be a triangular norm. A relation $R \in \mathcal{I} \mathcal{V} \mathcal{F} \mathcal{R}(X)$ is possibly $T$-transitive (pos- $T$-transitive), if

$$
T(\underline{R}(x, y), \underline{R}(y, z)) \leq \bar{R}(x, z) .
$$

This transitivity property is called possible $T$-transitivity which follows from the interpretation of the relation $\preceq$. Again, if $R(x, y)$ is an imprecise description of the relation between $x$ and $y$, and similarly for $R(y, z)$ and $R(x, z)$, then formula (3) expresses that it is possible to choose values in these intervals such that usual $T$-transitivity holds.

Proposition 4.3. Let $D \neq \emptyset$ and $R_{d} \in \mathcal{I V} \mathcal{F} \mathcal{R}(X), d \in D$. If $\left(R_{d}\right)$ is a family of pos-T-transitive relations, then the fuzzy relation $\bigwedge_{d \in D} R_{d}$ is pos-T-transitive.

P r o o f. If $R_{d}$ are pos- $T$-transitive relations, i.e., $T\left(\underline{R_{d}}(x, y), \underline{R_{d}}(y, z)\right) \leq \overline{R_{d}}(x, z)$, then by isotonicity of triangular norms, we know that min dominates any triangular norm $T$, i.e.,

$$
T\left(\bigwedge_{d \in D} \underline{R_{d}}(x, y), \bigwedge_{d \in D} \underline{R_{d}}(y, z)\right) \leq \bigwedge_{d \in D} T\left(\underline{R_{d}}(x, y), \underline{R_{d}}(y, z)\right) \leq \bigwedge_{d \in D} \overline{R_{d}}(x, z) .
$$

Proposition 4.4. Let $R \in \mathcal{I} \mathcal{V} \mathcal{F} \mathcal{R}(X)$. If $R$ is pos-T-transitive, then $R^{t}$ is also pos-T-transitive.

P r o o f. For an arbitrary $R \in \mathcal{I} \mathcal{V} \mathcal{F} \mathcal{R}(X)$ which is pos- $T$-transitive and by commutativity of a triangular norm we have

$$
\begin{aligned}
T\left(\underline{R^{t}}(x, y), \underline{R^{t}}(y, z)\right)= & T(\underline{R}(y, x), \underline{R}(z, y))= \\
& T(\underline{R}(z, y), \underline{R}(y, x)) \leq \bar{R}(z, x)=\overline{R^{t}}(x, z) .
\end{aligned}
$$

In the following theorems we use the fact (which follows from definition of pos- $T$-transitivity and definition of composition) that

LEMmA 4.5. Let $R \in \mathcal{I} \mathcal{V} \mathcal{F} \mathcal{R}(X) . R$ is pos-T-transitive if and only if $\underline{R}^{2} \leq \bar{R}$, where $\underline{R}^{2}=\underline{R} \circ_{T} \underline{R}$.

ThEOREM 4.6. Let $P, R \in \mathcal{I} \mathcal{V} \mathcal{F} \mathcal{R}(X)$. If $P, R$ are pos-T-transitive relations and $\underline{R} \circ_{T} \underline{P} \vee \underline{P} \circ_{T} \underline{R} \leq \bar{R} \vee \bar{P}$, then $R \vee S$ is pos-T-transitive.

P r o of. Let $P, R$ be interval-valued fuzzy pos- $T$-transitive relations. By Lemma 4.5, and the assumption $\underline{R} \circ_{T} \underline{P} \vee \underline{P} \circ_{T} \underline{R} \leq \bar{R} \vee \bar{P}$ and by Theorem 3.3 we have

$(\underline{R \vee P})^{2}=(\underline{R \vee P}){ }^{\circ}(\underline{R \vee P})=\underline{R}^{2} \vee \underline{R}{ }{ }_{T} \underline{P} \vee \underline{P} \circ_{T} \underline{R} \vee \underline{P^{2}} \leq \bar{R} \vee \bar{R} \vee \bar{P} \vee \bar{P}=\bar{R} \vee \bar{P}$, so $R \vee S$ is pos- $T$-transitive. 
TheOREM 4.7. Let $T_{1}, T_{2}, T_{1} \leq T_{2}$ be supremum preserving triangular norms $P, R \in \mathcal{I V \mathcal { F }}(X)$. If $P, R$ are pos- $T_{1}$-transitive and $\underline{R}{ }^{\circ}{ }_{T_{1}} \underline{P}=\underline{P}{ }^{\circ}{ }_{T_{1}} \underline{R}$, then $R \circ P$ is pos- $T_{1}$-transitive.

P r o of. Let $P, R$ be interval-valued fuzzy pos- $T$-transitive relations. By associativity of composition and the assumption $\underline{R} \circ_{T_{1}} \underline{P}=\underline{P}{ }^{\circ} T_{1} \underline{R}$, by Lemma 4.5 we have

$$
\begin{aligned}
\left(\underline{R \circ_{T_{1}} P}\right)^{2} & =\left(\underline{R} \circ_{T_{1}} \underline{P}\right)^{2}=\underline{R} \circ_{T_{1}}\left(\underline{P} \circ_{T_{1}} \underline{R}\right) \circ_{T_{1}} \underline{P}=\underline{R}^{2} \circ_{T_{1}} \underline{P}^{2} \\
& \leq \bar{R} \circ_{T_{1}} \bar{P} \leq \bar{R} \circ_{T_{2}} \bar{P} \\
& =\overline{P{ }^{\circ} T_{T_{2}} R} .
\end{aligned}
$$

Thus, $R \circ P$ is a pos- $T_{1}$-transitive relation.

CoRollaRY 4.8. Let $T_{1}, T_{2}, T_{1} \leq T_{2}$ be supremum preserving triangular norms and $R \in \mathcal{I V \mathcal { F R }}(X)$. If $R$ is pos-T-transitive, then $R^{n}$ is also pos-T-transitive.

Pr o of. By isotonicity of composition and powers, we obtain $\underline{R}^{n} \leq \bar{R}^{n-1}$, so $R^{n}$ preserves pos- $T_{1}$-transitivity.

Theorem 4.9. Let $R \in \mathcal{I} \mathcal{V} \mathcal{F} \mathcal{R}(X)$. If $\underline{R}$ is $T$-transitive, then $R$ is pos-T-transitive.

P r o o f. Let $\underline{R}$ be $T$-transitive. Then $\underline{R}^{2} \leq \underline{R} \leq \bar{R}$. Thus by Lemma 4.5 , we obtain pos- $T$-transitivity of $R$.

We also notice the connection between $\mathcal{T}$-transitivity and pos- $T$-transitivity.

Proposition 4.10. Let $R \in \mathcal{I} \mathcal{V} \mathcal{F} \mathcal{R}(X)$. If $R$ is $\mathcal{T}$-transitive, then $R$ is pos- $T_{1}$ -transitive.

Moreover, we know directly by definitions of $\mathcal{T}$-transitivity and composition, that

Proposition 4.11. Let $R \in \mathcal{I} \mathcal{V} \mathcal{F} \mathcal{R}(X), T_{1}, T_{2}, T_{1} \leq T_{2}$ be triangular norms. $R$ is $\mathcal{T}$-transitive if and only if $\underline{R}$ is $T_{1}$-transitive and $\bar{R}$ is $T_{2}$-transitive.

Moreover, we have the following property.

TheOREM 4.12. Let $T_{1}, T_{2}$ be triangular norms and $T_{1} \leq T_{2}$. If $R \in \mathcal{I} \mathcal{V F \mathcal { R }}(X)$ is pos- $T_{2}$-transitive, then $R$ is pos- $T_{1}$-transitive.

Proof. Let $R$ be pos- $T_{2}$-transitive. Then $T_{2}(\underline{R}(x, y), \underline{R}(y, z)) \leq \bar{R}(x, z)$ and by the fact that $T_{1} \leq T_{2}$ we have $T_{1}(\underline{R}(x, y), \underline{R}(y, z)) \leq T_{2}(\underline{R}(x, y), \underline{R}(y, z)) \leq$ $\bar{R}(x, z)$ for $x, y, z \in X$. As a result, $R$ is pos- $T_{1}$-transitive. 


\section{BARBARA PẸKALA — URSZULA BENTKOWSKA - BERNARD DE BAETS}

\section{Conclusion}

In future work, other operations for interval-valued fuzzy relations connected with the relation $\preceq$ may be considered. For example, a generalization of the here considered composition, i.e., sup $-A$ composition (where $A$ is an aggregation function) may be discussed. Moreover, other relations in the class of intervalvalued fuzzy relations may be studied, and as a consequence other types of transitivity, for example nec- $T$-transitivity.

\section{REFERENCES}

[1] BUSTINCE, H.-BURILLO, P.: Mathematical analysis of interval-valued fuzzy relations: application to approximate reasoning, Fuzzy Sets Syst. 113 (2000), 205-219.

[2] DE BAETS, B.-MESIAR, R.: Triangular norms on product lattices, Fuzzy Sets Syst. 104 (1999), 61-75.

[3] DE BAETS, B.: Aggregation of structured objects, Lecture during International Symposium on Aggregation on Bounded Lattices-ABLAT '14, Trabzon, Turkey, 2014, Karadeniz Technical University, 2014.

[4] DUBOIS, D.-PRADE, H.: Possibility theory. Plenum Press, New York, 1988.

[5] DUBOIS, D.- PRADE, H.: Gradualness, uncertainty and bipolarity: making sense of fuzzy sets, Fuzzy Sets Syst. 192 (2012), 3-24.

[6] DUBOIS, D.-GODO, L.-PRADE, H.: Weighted logics for artificial intelligence an introductory discussion, Int. J. Approx. Reason. 55 (2014), 1819-1829.

[7] FISHBURN, P. C.: Intransitive indifference with unequal indifference intervals, J. Math. Psychol. 7 (1970), 144-149.

[8] FISHBURN, P. C.: Utility Theory for Decision Making. J. Wiley, New York, 1970.

[9] FISHBURN, P. C.: Interval Orders and Interval Graphs. J. Wiley, New York, 1985.

[10] FODOR, J.-ROUBEnS, M.: Fuzzy Preference Modelling and Multicriteria Decision Support. Kluwer Acad. Publ., Dordrecht, 1994.

[11] GOGUEN, A.: L-fuzzy sets, J. Math. Anal. Appl. 18 (1967), 145-174.

[12] GONZÁLEZ DEL CAMPÓ, R.-GARMENDIA, L.-RECASENS, J.: Transitive closure of interval-valued relations, in: Proc. of the Joint Internat. Fuzzy Systems Assoc. World Congress and European Soc. of Fuzzy Logic and Technol. Conf.-IFSA/EUSFLAT '09 (J. P. Carvalho et al., eds.), Lisbon, Portugal, 2009, pp. 837-842.

[13] KARMAKAR, S.-BHUNIA, A. K.: A comparative study of different order relations of intervals, Reliab. Comput. 16 (2012), 38-72.

[14] PEुKALA, B.: Preservation of properties of interwal-valued fuzzy relations, in: Proc. of the Joint Internat. Fuzzy Systems Assoc. World Congress and European Soc. of Fuzzy Logic and Technol. Conf.-IFSA/EUSFLAT '09 (J. P. Carvalho et al., eds.), Lisbon, Portugal, 2009, pp. 1206-1210.

[15] SAMBUC, R.: Fonctions $\phi$-floues: Application Á l'aide au Diagnostic en Pathologie Thyroidienne, Ph.D. Thesis, Université de Marseille, France, 1975. (In French) 


\title{
COMPARABILITY RELATIONS IN CLASS OF INTERVAL-VALUED FUZZY RELATIONS
}

[16] ZADEH, L. A.: Fuzzy sets, Inform. Control 8 (1965), 338-353.

[17] ZADEH, L. A.: The concept of a linguistic variable and its application to approximate reasoning-I, Inform. Sci. 8 (1975), 199-249.

Received July 15, 2016

\author{
Barbara Pękala \\ Urszula Bentkowska \\ Interdisciplinary Centre for \\ Computational Modelling \\ University of Rzeszów \\ al. Rejtana $16 \mathrm{c}$ \\ PL-35-959 Rzeszów \\ POLAND \\ E-mail: bpekala@ur.edu.pl \\ ududziak@ur.edu.pl \\ Bernard De Baets \\ KERMIT \\ Research Unit Knowledge-based Systems \\ Ghent University \\ Coupure links 653 \\ B-9000 Gent \\ BELGIUM \\ E-mail: bernard.debaets@ugent.be
}

